\title{
Proof of Threshold Saturation for Spatially Coupled Sparse Superposition Codes
}

\author{
Jean Barbier, Member IEEE, Mohamad Dia and Nicolas Macris, Member IEEE. \\ Laboratoire de Théorie des Communications, Ecole Polytechnique Fédérale de Lausanne. \\ \{jean.barbier, mohamad.dia, nicolas.macris\}@epfl.ch
}

\begin{abstract}
Recently, a new class of codes, called sparse superposition or sparse regression codes, has been proposed for communication over the AWGN channel. It has been proven that they achieve capacity using power allocation and various forms of iterative decoding. Empirical evidence has also strongly suggested that the codes achieve capacity when spatial coupling and approximate message passing decoding are used, without need of power allocation. In this note we prove that state evolution (which tracks message passing) indeed saturates the potential threshold of the underlying code ensemble, which approaches in a proper limit the optimal threshold. Our proof uses ideas developed in the theory of low-density parity-check codes and compressive sensing.
\end{abstract}

\section{INTRODUCTION}

Sparse superposition (SS) codes introduced by Barron and Joseph for reliable communication over the additive white Gaussian noise channel (AWGNC) have been proven to approach capacity using power allocation and various efficient decoders [1-3]. An approximate message passing (AMP) decoder was introduced in [4], and the recent analysis [5] proves that this allows to reach capacity with the help of power allocation. Spatially coupled SS codes were introduced in $[6,7]$ and empirically shown to reach capacity under AMP without any need for power allocation. The empirical evidence shows that spatially coupled SS codes perform better than power allocated ones in the sense that they approach capacity faster in a proper limit [7]. Given this evidence, it is of interest to develop a rigorous theory for such coding constructions.

It is natural to address two conjectures. First, that spatially coupled SS codes allow to reach the so-called potential threshold of state evolution (SE). Second, that SE correctly tracks the AMP decoder. As we will argue, the potential threshold tends to capacity in a proper limit, so this would prove that the codes are capacity achieving.

The purpose of this note is to settle the first conjecture. We prove that for a general ensemble of spatially coupled coding matrices, the AMP threshold attains (in a suitable limit) the potential threshold of SE. This phenomenon is called threshold saturation. The precise statements of our main results are Theorem 5.7 and Corollary 5.8.

Threshold saturation was first established in the context of spatially coupled Low-Density Parity-Check codes for general binary input memoryless symmetric channels in [8], and is recognized as the basic mechanism underpinning the excellent performance of such codes [9]. It is interesting that essentially the same phenomenon can be established for a coding system operating on a channel with continuous inputs. This result is a stepping-stone towards establishing that spatially coupled SS codes achieve capacity on the AWGNC under AMP decoding. The remaining analysis to settle the second conjecture would require extending the one given for compressive sensing [10] to signals with correlated components in a spatially coupled system, as already done for the power allocated system [5].

To establish threshold saturation, we use the potential method along the lines of $[11,12]$ developed for LDPC and LDGM codes. Note that a similar (but different) potential to the one used here has been introduced in the context of scalar compressive sensing $[13,14]$. It is interesting that the potential method goes through for the present system involving a dense coding matrix and a fairly wide class of spatial couplings [15].

Coding constructions of the underlying and coupled ensembles are described in Sec. II. Sec. III reviews SE and potential formulations adapted to the present context. The AMP thresholds of underlying and coupled ensembles as well as the potential threshold are given precise definitions. Sec. IV introduces a notion of degradation and settles monotonicity properties of SE. The essential steps for the proof of threshold saturation are presented in Sec. V.

\section{CODE ENSEMBLES}

We first define the underlying and spatially coupled ensembles of SS codes for transmission over an AWGNC. We will often use the shorter notations $\left[a_{1}: a_{n}\right]$ and $\left\{b_{1}: b_{n}\right\}$ instead of $\left[a_{1}, \ldots, a_{n}\right]$ and $\left\{b_{1}, \ldots, b_{n}\right\}$ for $n$-tuples.

\section{A. The underlying ensemble}

In the framework of SS codes, the information word $\mathbf{s}=$ $\left[\mathbf{s}_{1}: \mathbf{s}_{L}\right]$ is a vector made of $L$ sections. Each section $\mathbf{s}_{l}, l \in$ $\{1: L\}$, is a $B$-dimensional vector with one component equal to 1 and $B-1$ components equal to 0 . For example if ( $B=$ $3, L=4)$, a valid information word is $\mathbf{s}=[001,010,100,010]$. We call $B$ the section size (or alphabet size) and set $N=$ $L B$. A codeword Fs $\in \mathbb{R}^{M}$ is generated from a fixed coding matrix $\mathbf{F} \in \mathbb{R}^{M \times N}$. We consider random codes generated by $\mathbf{F}$ drawn from the ensemble of random matrices with i.i.d real Gaussian entries with distribution $\mathcal{N}(0,1 / L)$. The cardinality of the code is $B^{L}$, the block length is $M$, and the (design) rate is $R=L \log _{2}(B) / M=N \log _{2}(B) /(M B)$. The code is thus specified by the basic parameters $(M, R, B)$. 


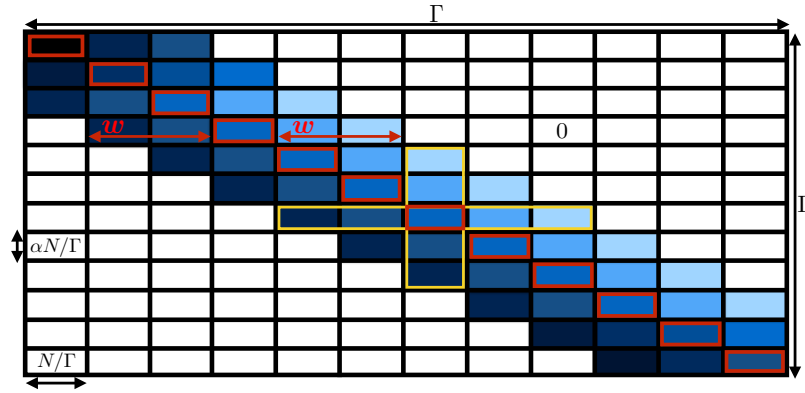

Fig. 1. A spatially coupled coding matrix $\in \mathbb{R}^{M \times N}$ made of $\Gamma \times \Gamma$ blocks indexed by $(r, c)$, each with $N / \Gamma$ columns and $M / \Gamma=\alpha N / \Gamma$ rows. The i.i.d elements in block $(r, c)$ have distribution $\mathcal{N}\left(0, J_{r, c} / L\right)$. Away from the boundaries, in addition to the diagonal (in red), there are $w$ forward and backward coupling blocks. In this example, the design function $g_{w}$ enforces a stronger backward coupling. Blocks are darker at the boundaries because the variances are larger so as to enforce the variance normalization $\sum_{c=1}^{\Gamma} J_{r, c} / \Gamma=1 \forall r$. The yellow shape emphasizes variance symmetry $\sum_{r=1}^{\mathrm{f}=1} J_{r, k} / \Gamma=\sum_{c=1}^{\Gamma} J_{k, c} / \Gamma=1$ verified if $k \in\{2 w+1: \Gamma-2 w\}$.

Codewords are transmitted through an AWGNC, i.e., the received signal is $\mathbf{y}=\mathbf{F s}+\boldsymbol{\xi}$ where $\xi_{\mu} \sim \mathcal{N}\left(0, \sigma^{2}\right) \forall \mu$. Power is normalized thanks to the $1 / L$ variance of the entries of $\mathbf{F}$, so that the signal-to-noise ratio is $\operatorname{snr}=1 / \sigma^{2}$.

The decoding task is to infer the information word $\mathbf{s}$ from channel observations $\mathbf{y}$. This is obviously very similar in spirit to compressive sensing, where one wants to infer a sparse signal from a certain number of measurements. For this reason, our language and techniques are sometimes borrowed from compressive sensing. In particular, the code rate can be linked to a "measurement rate" $\alpha:=M / N=\log _{2}(B) /(B R)$.

\section{B. The spatially coupled ensemble}

The construction has similarities with [16]. We consider spatially coupled codes based on coding matrices in $\mathbb{R}^{M \times N}$ as described in details in Fig. 1. This ensemble of matrices is parametrized by $\left(M, R, B, \Gamma, w, g_{w}\right)$, where $w$ is the coupling window and $g_{w}$ is the design function. This is any function verifying $g_{w}(x)=0$ if $|x|>1$ and $g_{w}(x) \geq g_{0}>0$ else, which is Lipschitz continuous on its support with Lipschitz constant $g_{*}$. The constants $g_{0}, g_{*}$ are independent of $w$. Moreover, we impose the normalization $\sum_{k=-w}^{w} g_{w}(k / w) /(2 w+1)=1$.

From $g_{w}$, we construct the variances of the blocks: the i.i.d entries inside the block $(r, c)$ are distributed as $\mathcal{N}\left(0, J_{r, c} / L\right)$, where $J_{r, c}:=\gamma_{r} \Gamma g_{w}((r-c) / w) /(2 w+1)$. Here $\gamma_{r}$ enforces the variance normalization $\sum_{c=1}^{\Gamma} J_{r, c} / \Gamma=1 \forall r$. This implies (by the law of large numbers) that $[\mathbf{F s}]_{\mu}^{2} \rightarrow 1 \forall \mu$ as $L \rightarrow \infty$, i.e. the asymptotic power $\lim _{L \rightarrow \infty} \sum_{\mu=1}^{M}[\mathbf{F s}]_{\mu}^{2} / M=1$, and the $\mathrm{snr}=1 / \sigma^{2}$ is homogeneous.

The spatial coupling induces a natural decomposition of the signal into $\Gamma$ blocks (associated with the block-columns of the matrix), each made of $L / \Gamma$ sections. One key element of spatially coupled codes is the seed introduced at the boundaries. We assume the sections in the first and last $4 w$ blocks of the information word $\mathbf{s}$ to be known by the decoder. This boundary condition can be interpreted as perfect side measurements that propagate inward and boost the performance. The seed induces a rate loss in the effective rate of the code, $R_{\text {eff }}=R\left(1-\frac{8 w}{\Gamma}\right)$. However, this loss vanishes as $\Gamma \rightarrow \infty$.

\section{State EVOlution AND POTENTIAL FORMULATION}

We now give the state evolution associated to the underlying and spatially coupled ensembles, which is conjectured to track the performance of the AMP decoder. We then define an appropriate potential function for each ensemble.

\section{A. State evolution}

The goal is to iteratively compute the mean square error (MSE) $\tilde{E}^{(t)}=\frac{1}{L} \mathbb{E}_{\mathbf{s}, \mathbf{y}}\left[\sum_{j=1}^{N}\left(\hat{s}_{j}^{(t)}-s_{j}\right)^{2}\right]$ of the AMP estimates $\left\{\hat{s}_{j}^{(t)}\right\}$ at iteration $t \in \mathbb{N}$. We need a few definitions to express the iterations. Let $\Sigma(E):=\sqrt{R\left(\sigma^{2}+E\right)}$. We define a $d e$ noiser $f_{i}(\Sigma(E))$ as the minimum mean square error (MMSE) estimator of the $i$-th component of a section sent through an effective AWGNC with a noise $\mathcal{N}\left(0, \Sigma(E)^{2} / \log _{2}(B)\right)$, when the input signal is uniformly distributed. Explicitly, if $\mathbf{z}=\left[z_{1}: z_{B}\right]$ denote $B$ independent standardized Gaussian random variables (with zero mean and unit variance), and $p_{0}(\mathbf{x})=\frac{1}{B} \sum_{i=1}^{B} \delta_{x_{i}, 1} \prod_{k \neq i}^{B} \delta_{x_{k}, 0}$, we set for $i=1: B$

$$
\begin{aligned}
& f_{i}(\Sigma(E)):=\frac{\sum_{\mathbf{x}} e^{-\frac{\| \mathbf{x}-\left(\mathbf{s}+\mathbf{x} \Sigma(E) / \sqrt{\left.\log _{2}(B)\right)} \|_{2}^{2}\right.}{2 \Sigma(E)^{2} / \log _{2}(B)}} p_{0}(\mathbf{x}) x_{i}}{\sum_{\mathbf{x}} e^{-\frac{\| \mathbf{x}-\left(\mathbf{s}+\Sigma \Sigma(E) / \sqrt{\left.\log _{2}(B)\right)}\right.}{2 \Sigma(E)^{2} / \log _{2}(B)}} p_{0}(\mathbf{x})} \\
& =\left[1+\sum_{k \neq i}^{B} e^{\left(s_{k}-s_{i}\right) \log _{2}(B) / \Sigma(E)^{2}+\left(z_{k}-z_{i}\right) \sqrt{\log _{2}(B) / \Sigma(E)}}\right]^{-1} .
\end{aligned}
$$

Note that the denoiser also depends on $\mathbf{s}, \mathbf{z}$. Furthermore, we define the SE operator of the underlying system as

$$
T_{\mathbf{u}}(E):=\mathbb{E}_{\mathbf{s}, \mathbf{z}}\left[\sum_{i=1}^{B}\left(f_{i}(\Sigma(E))-s_{i}\right)^{2}\right] .
$$

This is nothing else than the MSE associated to the MMSE estimator of the effective AWGNC with noise $\mathcal{N}\left(0, \Sigma(E)^{2} / \log _{2}(B)\right)$. The SE iterations for the underlying system's MSE can now be expressed as

$$
\tilde{E}^{(t+1)}=T_{\mathrm{u}}\left(\tilde{E}^{(t)}\right), \quad t \geq 0 .
$$

To track the performance of the AMP decoder the iterations are initialized with $\tilde{E}^{(0)}=1$. The monotonicity and boundedness of the iterations of SE, discussed in Sec. IV, ensure that actually all initial conditions reach a fixed point.

Definition 3.1 (MSE Floor): The MSE floor $E_{0}$ is defined as the fixed point reached from the initial condition $\tilde{E}^{(0)}=0$. In other words $E_{0}=T_{\mathrm{u}}^{(\infty)}(0)$.

Definition 3.2 (Bassin of attraction): The basin of attraction of the MSE floor $E_{0}$ is $\mathcal{V}_{0}:=\left\{E \mid T_{\mathrm{u}}^{(\infty)}(E)=E_{0}\right\}$.

Definition 3.3 (Threshold of underlying ensemble): The AMP threshold is $R_{\mathrm{u}}:=\sup \left\{R>0 \mid T_{\mathrm{u}}^{(\infty)}(1)=E_{0}\right\}$.

It can be shown that for the present system $T_{\mathrm{u}}^{(\infty)}(0)$ and $T_{\mathrm{u}}^{(\infty)}(1)$ are the only two possible fixed points. For $R<R_{\mathrm{u}}$, there is only one fixed point, namely the "good" one $T_{\mathrm{u}}^{(\infty)}(0)$, and for large section size $B$ the MSE floor and section error 
rate are small. Instead if $R>R_{\mathrm{u}}$, the decoder is blocked by the "bad" fixed point $T_{\mathrm{u}}^{(\infty)}(1) \neq E_{0}$ and AMP cannot decode.

We now turn our attention to SE for the spatially coupled system. The perfomance of AMP is described by an MSE profile $\left\{\tilde{E}_{c} \mid c=1\right.$ : $\left.\Gamma\right\}$ along the "spatial dimension". Since we assume that the boundary sections are known to the decoder, we enforce the pinning condition $\tilde{E}_{c}=0$ for $c \in\{1: 4 w\} \cup\{\Gamma-4 w+1: \Gamma\}$. For $c$ not in this set, by definition $\tilde{E}_{c}:=\frac{\Gamma}{L} \sum_{l \in c} \mathbb{E}_{\mathbf{s}, \mathbf{y}}\left[\left\|\hat{\mathbf{s}}_{l}-\mathbf{s}_{l}\right\|_{2}^{2}\right]$, where the sum $l \in c$ is over the set of indices of the $L / \Gamma$ sections composing the $c$-th block of $\mathbf{s}$. It is more convenient to work with a smoothed error profile (referred as the error profile) $\mathbf{E}=\left\{E_{r} \mid r=1: \Gamma\right\}, E_{r}:=\frac{1}{\Gamma} \sum_{c=1}^{\Gamma} J_{r, c} \tilde{E}_{c}$. Indeed, this change of variables makes the problem mathematically more tractable for spatially coupled codes. The pinning condition becomes $E_{r}=0$ for $r \in \mathcal{A}:=\{1: 3 w\} \cup\{\Gamma-3 w+1: \Gamma\}$.

We define an effective noise for block $c \in\{1: \Gamma\}$,

$$
\Sigma_{c}(\mathbf{E}):=\left[\frac{1}{\Gamma} \sum_{r=1}^{\Gamma} \frac{J_{r, c}}{R\left(\sigma^{2}+E_{r}\right)}\right]^{-1 / 2}
$$

and the coupled SE operator

$$
\left[T_{\mathrm{c}}(\mathbf{E})\right]_{r}=\frac{1}{\Gamma} \sum_{c=1}^{\Gamma} J_{r, c} \mathbb{E}_{\mathbf{s}, \mathbf{z}}\left[\sum_{i=1}^{B}\left(f_{i}\left(\Sigma_{c}(\mathbf{E})\right)-s_{i}\right)^{2}\right] .
$$

$T_{\mathrm{c}}(\mathbf{E})$ is vector valued and here we have written its $r$-th component. The SE iterations then read for $r \notin \mathcal{A}$

$$
E_{r}^{(t+1)}=\left[T_{\mathrm{c}}\left(\mathbf{E}^{(t)}\right)\right]_{r}, \quad t \geq 0 .
$$

For $r \in \mathcal{A}$, the pinning condition $E_{r}^{(t)}=0$ is enforced at all times. SE is initialized with $E_{r}^{(0)}=1$ for $r \notin \mathcal{A}$.

Let $\mathbf{E}_{0}:=\left[E_{r}=E_{0}, r=1: \Gamma\right]$ be the MSE floor profile.

Definition 3.4 (Threshold of coupled ensemble): The AMP threshold of the coupled system is defined as $R_{\mathrm{c}}:=$ $\liminf _{\Gamma, w \rightarrow \infty} \sup \left\{R>0 \mid T_{\mathrm{c}}^{(\infty)}(\mathbf{1}) \prec \mathbf{E}_{0}\right\}$ where $\mathbf{1}$ is the all ones vector. Here the $\liminf \operatorname{in}_{\Gamma, w \rightarrow \infty}$ is taken along sequences where first $\Gamma \rightarrow \infty$ and then $w \rightarrow \infty$.

\section{B. Potential formulation}

The fixed point equations associated to SE iterations can be reformulated as stationary point equations for suitable potential functions. These are in general not unique. However, the "correct" guess (i.e. the one that allows to prove full threshold saturation) can be derived by the replica method of statistical physics [4] .

The potential of the underlying ensemble is $F_{\mathrm{u}}(E):=$ $U_{\mathrm{u}}(E)-S_{\mathrm{u}}(\Sigma(E))$, with

$$
\left\{\begin{array}{l}
U_{\mathrm{u}}(E):=\frac{1}{2 R} \log _{2}\left(\left(\sigma^{2}+E\right) e^{-\frac{E}{\sigma^{2}+E}}\right), \\
S_{\mathrm{u}}(\Sigma(E)):=\mathbb{E}_{\mathbf{z}}\left[\log _{B}\left(1+\sum_{i=2}^{B} e_{i}(\Sigma(E))\right)\right],
\end{array}\right.
$$

where $e_{i}(x):=\exp \left(\left(z_{i}-z_{1}\right) \sqrt{\log _{2}(B)} / x-\log _{2}(B) / x^{2}\right)$.

The potential of the spatially coupled ensemble is $F_{\mathrm{c}}(\mathbf{E})=$ $U_{\mathrm{c}}(\mathbf{E})-S_{\mathrm{c}}(\mathbf{E})$ where

$$
\left\{\begin{array}{l}
U_{\mathrm{c}}(\mathbf{E}):=\sum_{r=1}^{\Gamma} U_{\mathrm{u}}\left(E_{r}\right) \\
S_{\mathrm{c}}(\mathbf{E}):=\sum_{c=1}^{\Gamma} S_{\mathrm{u}}\left(\Sigma_{c}(\mathbf{E})\right) .
\end{array}\right.
$$

Let us pause for an instant and give the statistical physics interpretation of these formulas. The posterior distribution $p(\mathbf{s} \mid \mathbf{y})=\exp \left(-\|\mathbf{y}-\mathbf{F s}\|_{2}^{2} / 2 \sigma^{2}\right) p_{0}(\mathbf{s}) / Z$ ( $Z$ the normalizing factor) can be interpreted as the Gibbs distribution of a disordered spin system (s being the annealed "spin" degrees of freedom, $\mathbf{y}$ and $\mathbf{F}$ the quenched "disorder"). The potential functions $F$ are "Bethe free energies" averaged over the disorder. They are equal to "energy" terms $U$ minus "entropy" terms $S$. One can prove that both terms are increasing in $\Sigma$. This is "physically" natural if the effective channel noise $\Sigma$ is interpreted as a kind of effective "temperature".

Definition 3.5 (Free energy gap): The free energy gap is $\Delta F_{\mathrm{u}}:=\inf _{E \notin \mathcal{V}_{0}}\left(F_{\mathrm{u}}(E)-F_{\mathrm{u}}\left(E_{0}\right)\right)$, with the convention that the infimum over the empty set is $\infty$ (this happens for $R<$ $R_{\mathrm{u}}$ ).

Definition 3.6 (Potential threshold): The potential threshold is $R_{\text {pot }}:=\sup \left\{R>0 \mid \Delta F_{\mathrm{u}}>0\right\}$.

The connection between these potentials and SE is given by the following Lemma.

Lemma 3.7: If $\stackrel{\circ}{E}$ is a fixed point of (2), i.e., $T_{\mathrm{u}}(\stackrel{\circ}{E})=\stackrel{\circ}{E}$, then $\left[\frac{\partial F_{\mathbf{u}}}{\partial E}\right]_{E}^{\circ}=0$. Similarly for the coupled system, if $\stackrel{\circ}{\mathbf{E}}$ is a fixed point of (5) then $\left[\frac{\partial F_{\mathrm{c}}}{\partial E_{r}}\right]_{\mathbf{E}}=0 \forall r \in\{3 w+1: \Gamma-3 w\}$.

The proof is technical and we skip it here. Let us just indicate that it proceeds by computing the derivatives of the potentials, uses Gaussian integration by parts formulas and channel symmetry. Similar results can be found in $[11,12]$.

It is noteworthy that what is called a "Bethe entropy" in the statistical physics literature, has an information theoretic interpretation, and is actually a Shannon conditionnal entropy for an effective channel. Let $\mathbf{X}$ be a $B$-dimensional random vector with distribution $p_{0}(\mathbf{x})=\frac{1}{B} \sum_{i=1}^{B} \delta_{x_{i}, 1} \prod_{k \neq i}^{B} \delta_{x_{k}, 0}$. Take the output $\mathbf{Y}$ of an AWGNC with i.i.d noise $\mathcal{N}\left(0, \Sigma^{2} / \log _{2}(B)\right)$ for each component, when the input is $\mathbf{X}$. Then it is an exercise to check that $H(\mathbf{X} \mid \mathbf{Y})=S_{\mathrm{u}}(\Sigma) \log _{2}(B)$. This identification clearly shows that $S_{\mathrm{u}}(\Sigma)$ must be an increasing function of $\Sigma$. Also, it allows to give information theoretic expressions for the potential functions. We note that such expressions have already been written down for scalar compressive sensing $[13,14]$.

We also point out that there is another way to derive potential functions by integrating out the SE fixed point equations after premultiplication by an "integrating factor". When the correct "integrating factor" is used, one recovers the information theoretic expressions of the potential functions. This method is discussed in [14] for a wide range of problems including scalar compressive sensing, and one can check it extends to the present setting. A key point for this method is the well known relation between mutual information (or conditional entropy) and MMSE [17]. Here this relation takes the form $\frac{d H(\mathbf{X} \mid \mathbf{Y})}{d\left(\Sigma^{-2}\right)}=-\frac{1}{2} \operatorname{mmse}(\Sigma)$ (here mmse $=T_{\mathrm{u}}$ where $T_{\mathrm{u}}$ is the r.h.s of (1) viewed as a function of $\Sigma$ ).

\section{Large B analysis and connection with Shannon's capacity}

Let us now emphasize the connection between the potential threshold $R_{\text {pot }}$ and Shannon's capacity $C=\frac{1}{2} \log _{2}(1+\mathrm{snr})$. 
The large section size limit of the potential of the underlying system becomes [18]

$$
\lim _{B \rightarrow \infty} F_{\mathrm{u}}(E)=U_{\mathrm{u}}(E)-\max \left(0,1-\frac{1}{2 \ln (2) \Sigma(E)^{2}}\right),
$$

(where we recall $\Sigma(E)^{2}:=R\left(\sigma^{2}+E\right)$ ). The analysis of this function of $E \in[0,1]$ shows the following. For $R<$ $\left[\left(1+\sigma^{2}\right) 2 \ln (2)\right]^{-1}$ there is a unique minimum at $E=0$. For $\left[\left(1+\sigma^{2}\right) 2 \ln (2)\right]^{-1}<R<C, E=0$ is the global minimum but there exists a local minimum at $E=1$. When $R>C$ the global minimum is at $E=1$ and $E=0$ is a local minimum. Therefore we can identify $\lim _{B \rightarrow \infty} R_{\text {pot }}=C$ and $\lim _{B \rightarrow \infty} R_{\mathrm{u}}=\left[\left(1+\sigma^{2}\right) 2 \ln (2)\right]^{-1}$.

Let us also point out that these are static properties of the system which are independent of the decoding algorithms. In a sense they confirm in an alternative way that the codes must achieve capacity under optimal decoding [19].

\section{Properties of the Coupled System}

Monotonicity properties of the SE operators $T_{\mathrm{u}}$ and $T_{\mathrm{c}}$ are key elements in the analysis. We start by defining a suitable notion of degradation.

Definition 4.1 (Degradation): A profile $\mathbf{E}$ is degraded (resp. strictly degraded) with respect to another one $\mathbf{G}$, denoted as $\mathbf{E} \succeq \mathbf{G}$ (resp. $\mathbf{E} \succ \mathbf{G}$ ), if $E_{r} \geq G_{r} \forall r$ (resp. if $\mathbf{E} \succeq \mathbf{G}$ and there exists some $r$ such that $E_{r}>G_{r}$ ).

Lemma 4.2: The SE operator of the coupled system maintains degradation in space, i.e., if $\mathbf{E} \succeq \mathbf{G}$, then $T_{\mathfrak{c}}(\mathbf{E}) \succeq T_{\mathfrak{c}}(\mathbf{G})$. This property is verified by $T_{\mathrm{u}}$ for a scalar error as well.

Proof: From (3) it is immediately seen that $\mathbf{E} \succeq \mathbf{G}$ implies $\Sigma_{c}(\mathbf{E}) \geq \Sigma_{c}(\mathbf{G}) \forall c$. Now, the SE operator (4) can be interpreted as an average over the spatial dimension of local MMSE's. The local MMSE's are the ones of $B$-dimensional AWGN channels with effective noise $\mathcal{N}\left(0, \Sigma_{c}^{2} / \log _{2}(B)\right)$. The MMSE's are increasing functions of $\Sigma_{c}^{2}$ : this is intuitively clear but we provide an explicit formula for the derivative below. Thus $\left[T_{\mathrm{c}}(\mathbf{E})\right]_{r} \geq\left[T_{\mathfrak{c}}(\mathbf{G})\right]_{r} \forall r$, which means $T_{\mathrm{c}}(\mathbf{E}) \succeq T_{\mathrm{c}}(\mathbf{G})$.

The derivative of the MMSE of the Gaussian channel with i.i.d noise $\mathcal{N}\left(0, \Sigma^{2}\right)$ can be computed as

$$
\frac{d \text { mmse }(\Sigma)}{d\left(\Sigma^{-2}\right)}=-2 \mathbb{E}_{\mathbf{X}, \mathbf{Y}}\left[\|\mathbf{X}-\mathbb{E}[\mathbf{X} \mid \mathbf{Y}]\|_{2}^{2} \operatorname{Var}[\mathbf{X} \mid \mathbf{Y}]\right]
$$

This formula is valid for vector distributions $p_{0}(\mathbf{x})$, and in particular, for our $B$-dimensional sections. It explicitly confirms that $T_{\mathrm{u}}\left(\right.$ resp. $\left.\left[T_{\mathrm{c}}\right]_{r}\right)$ is an increasing function of $\Sigma\left(\right.$ resp. $\left.\Sigma_{c}\right)$.

Corollary 4.3: The SE operator of the coupled system maintains degradation in time, i.e., $T_{\mathrm{c}}\left(\mathbf{E}^{(t)}\right) \preceq \mathbf{E}^{(t)}$, implies $T_{\mathrm{c}}\left(\mathbf{E}^{(t+1)}\right) \preceq \mathbf{E}^{(t+1)}$. Similarly $T_{\mathrm{c}}\left(\mathbf{E}^{(t)}\right) \succeq \mathbf{E}^{(t)}$ implies $T_{\mathrm{c}}\left(\mathbf{E}^{(t+1)}\right) \succeq \mathbf{E}^{(t+1)}$. Furthermore, the limiting error profile $\mathbf{E}^{(\infty)}:=T_{\mathrm{c}}^{(\infty)}\left(\mathbf{E}^{(0)}\right)$ exists. This property is verified by $T_{\mathrm{u}}$ for the underlying system as well.

Proof: The degradation statements are a consequence of Lemma 4.2. The existence of the limits follows from the monotonicity of the operator and boundedness of the MSE.

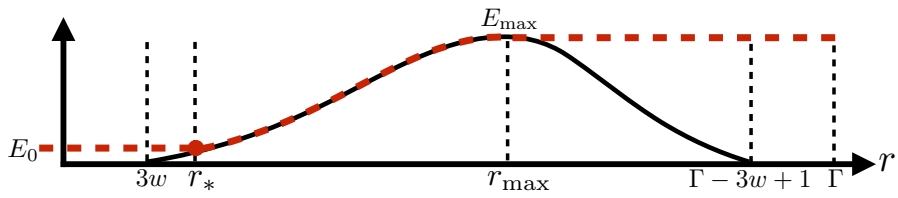

Fig. 2. A profile $\mathbf{E}^{*}$ (solid) and its associated saturated non decreasing profile $\mathbf{E}$ (dashed). The former has a plateau at 0 for all $r \leq 3 w$ and it increases until $r_{\max }$ where it reaches its maximum value $E_{\max }$. Then it decreases to 0 at $\Gamma-3 w+1$ and remains null after. The saturated profile starts with a plateau at $E_{0}$ for all $r \leq r_{*}$, where $r_{*}$ is the position defined by $E_{r_{*}}^{*}=E_{0}$, and then matches $\mathbf{E}^{*}$ for all $r \in\left\{r_{*}: r_{\max }\right\}$. It then saturates to $E_{\max }$ for all $r \geq r_{\max }$. By construction, $\mathbf{E}$ is non decreasing and verifies $\mathbf{E} \succ \mathbf{E}^{*}$.

\section{PRoOf OF THRESHOLD SATURATION}

The pinning step together with the monotonicity properties of the coupled SE imply that the fixed point profile $\mathbf{E}^{*}$ must adopt a shape similar to Fig. 2 (note $E_{\max } \in[0,1]$ ). We associate to $\mathbf{E}^{*}$ a saturated profile denoted $\mathbf{E}$. Its construction is described in detail in Fig. 2. The saturated profile $\mathbf{E}$ is strictly degraded with respect to $\mathbf{E}^{*}$, thus $\mathbf{E}$ serves as an upper bound in our proof.

\section{A. Coupled potential change evaluation by Taylor expansion}

Definition 5.1 (Shift operator): The shift operator is defined pointwise as $[\mathbf{S}(\mathbf{E})]_{1}:=E_{0},[\mathbf{S}(\mathbf{E})]_{r}:=E_{r-1}$.

Lemma 5.2: Let $\mathbf{E}$ be a saturated profile. If $\hat{\mathbf{E}}:=(1-\hat{t}) \mathbf{E}+$ $\hat{t} \mathbf{S}(\mathbf{E})$ and $\Delta E_{r}:=E_{r}-E_{r-1}$, then for a proper $\hat{t} \in[0,1]$,

$$
F_{\mathrm{c}}(\mathrm{S}(\mathbf{E}))-F_{\mathrm{c}}(\mathbf{E})=\frac{1}{2} \sum_{r, r^{\prime}=1}^{\Gamma} \Delta E_{r} \Delta E_{r^{\prime}}\left[\frac{\partial^{2} F_{\mathrm{c}}}{\partial E_{r} \partial E_{r^{\prime}}}\right]_{\hat{\mathbf{E}}} .
$$

Proof: $F_{\mathrm{c}}(\mathrm{S}(\mathbf{E}))-F_{\mathrm{c}}(\mathbf{E})$ can be expressed as

$$
\frac{1}{2} \sum_{r, r^{\prime}=1}^{\Gamma} \Delta E_{r} \Delta E_{r^{\prime}}\left[\frac{\partial^{2} F_{\mathrm{c}}}{\partial E_{r} \partial E_{r^{\prime}}}\right]_{\hat{\mathbf{E}}}-\sum_{r=1}^{\Gamma} \Delta E_{r}\left[\frac{\partial F_{\mathrm{c}}}{\partial E_{r}}\right]_{\mathbf{E}} .
$$

By saturation of $\mathbf{E}, \Delta E_{r}=0 \forall r \in \mathcal{B}:=\left\{1: r_{*}\right\} \cup\left\{r_{\max }+\right.$ $1: \Gamma\}$. Moreover for $r \notin \mathcal{B}, E_{r}=\left[T_{c}(\mathbf{E})\right]_{r}$, and thus by Lemma 3.7 the potential derivative cancels at these positions. Hence the last sum in (10) cancels.

Lemma 5.3: The saturated profile $\mathbf{E}$ is smooth, i.e. $\left|\Delta E_{r}\right|<$ $\left(g_{*}+\tilde{g}\right) / w$ where $\tilde{g}:=\max \left(1+g_{*}, g_{0}+2 g_{*}\right)$. Recall $g_{0}$ and $g_{*}$ are independent of $w$ and $\Gamma$.

Proof: $\Delta E_{r}=0$ for all $r \in \mathcal{B}$. By construction of $\left\{J_{r, c}\right\}$ and using Lipschitz continuity of $g_{w}$, we have for all $r \notin \mathcal{B}$ that $\left|\Delta E_{r}\right|=\left|\sum_{c}\left(J_{r, c}-J_{r-1, c}\right) \tilde{E}_{c}\right| / \Gamma<\left(g_{*}+\tilde{g}\right) / w$.

Lemma 5.4: The coupled potential verifies

$$
\frac{1}{2}\left|\sum_{r, r^{\prime}=1}^{\Gamma} \Delta E_{r} \Delta E_{r^{\prime}}\left[\frac{\partial^{2} F_{\mathbf{c}}}{\partial E_{r} \partial E_{r^{\prime}}}\right]_{\hat{\mathbf{E}}}\right|<K / w,
$$

where $K$ is independent of $w$ and $\Gamma$.

The proof uses Lemma 5.3 and the monotonicity of $E_{r}$. Bounding the Hessian is rather long but does not present major difficulties. 


\section{B. Direct evaluation of the coupled potential change}

Lemma 5.5: Let $\mathbf{E}$ be a saturated profile such that $\mathbf{E} \succ \mathbf{E}_{0}$. Then $E_{\max } \notin \mathcal{V}_{0}$.

Proof: The non decreasing error profile and the assumption that $\mathbf{E} \succ \mathbf{E}_{0}$ imply that $E_{\max }>E_{0}$. Moreover, $E_{\max } \leq$ $\left[T_{\mathrm{c}}(\mathbf{E})\right]_{r_{\max }} \leq T_{\mathrm{s}}\left(E_{\max }\right)$ where the first inequality follows from $\mathbf{E} \succ \mathbf{E}^{*}$ and the monotonicity of $T_{\mathrm{c}}$, while the second comes from the variance symmetry at $r_{\max }$ and the fact that $\mathbf{E}$ is non decreasing. Combining these with the monotonicity of $T_{\mathrm{s}}$ gives: $T_{\mathrm{s}}\left(E_{\max }\right) \geq E_{\max } \Rightarrow T_{\mathrm{s}}^{(\infty)}\left(E_{\max }\right) \geq E_{\max }>$ $E_{0} \Rightarrow E_{\max } \notin \mathcal{V}_{0}$.

Lemma 5.6: Let $\mathbf{E}$ be a saturated profile such that $\mathbf{E} \succ \mathbf{E}_{0}$. Then $F_{\mathrm{c}}(\mathrm{S}(\mathbf{E}))-F_{\mathrm{c}}(\mathbf{E}) \leq-\Delta F_{\mathrm{u}}$.

Proof: The contribution of the change in the energy term is a perfect telescoping sum:

$$
U_{\mathrm{c}}(\mathbf{S}(\mathbf{E}))-U_{\mathrm{c}}(\mathbf{E})=U_{\mathrm{u}}\left(E_{0}\right)-U_{\mathrm{u}}\left(E_{\max }\right) .
$$

We now deal with the contribution of the change in the entropy term. We first notice that, using the variance symmetry, $\Sigma_{c}(\mathbf{E})=\Sigma_{c+1}(\mathbf{S}(\mathbf{E})) \forall c \in\{2 w+1: \Gamma-2 w-1\}$, which makes the sum telescoping in this set. Thus

$$
\begin{aligned}
& S_{\mathrm{c}}(\mathbf{E})-S_{\mathrm{c}}(\mathbf{S}(\mathbf{E}))=S_{\mathrm{u}}\left(\Sigma_{\Gamma-2 w}(\mathbf{E})\right)-S_{\mathrm{u}}\left(\Sigma_{2 w+1}(\mathbf{S}(\mathbf{E}))\right) \\
& -\sum_{c \in \mathcal{S}}\left[S_{\mathrm{u}}\left(\Sigma_{c}(\mathbf{S}(\mathbf{E}))\right)-S_{\mathrm{u}}\left(\Sigma_{c}(\mathbf{E})\right)\right]
\end{aligned}
$$

where $\mathcal{S}:=\{1: 2 w\} \cup\{\Gamma-2 w+1: \Gamma\}$. By saturation, $\mathbf{E}$ possesses the following property: $[\mathbf{S}(\mathbf{E})]_{r}=[\mathbf{E}]_{r} \forall r \in$ $\left\{1: r_{*}\right\} \cup\left\{r_{\max }+1: \Gamma\right\} \Rightarrow \Sigma_{c}(\mathbf{S}(\mathbf{E}))=\Sigma_{c}(\mathbf{E}) \forall c \in$ $\mathcal{S}$ and thus the sum in (13) cancels. Furthermore, one can show, using the saturation of $\mathbf{E}$ and the variance symmetry, that $\Sigma_{2 w+1}(\mathbf{S}(\mathbf{E}))=\Sigma\left(E_{0}\right)$. Using the same arguments, in addition to the fact that $\mathbf{E} \succ \mathbf{E}_{0} \Rightarrow r_{\max } \leq \Gamma-3 w$, we obtain $\Sigma_{\Gamma-2 w}(\mathbf{E})=\Sigma\left(E_{\max }\right)$. Hence, (13) yields the following:

$$
S_{\mathrm{c}}(\mathbf{E})-S_{\mathrm{c}}(\mathrm{S}(\mathbf{E}))=S_{\mathrm{u}}\left(\Sigma\left(E_{\max }\right)\right)-S_{\mathrm{u}}\left(\Sigma\left(E_{0}\right)\right) .
$$

Combining (12) with (14) and using Lemma 5.5 gives

$$
F_{\mathrm{c}}(\mathrm{S}(\mathbf{E}))-F_{\mathrm{c}}(\mathbf{E})=-F_{\mathrm{u}}\left(E_{\max }\right)+F_{\mathrm{u}}\left(E_{0}\right) \leq-\Delta F_{\mathrm{u}} .
$$

Theorem 5.7: Assume a spatially coupled SS code ensemble is used for communication through an AWGNC. Fix $R<R_{\text {pot }}$, $w>K / \Delta F_{\mathrm{u}}(K$ is independent of $w$ and $\Gamma)$ and $\Gamma>8 w$ (such that the code is well defined). Then any fixed point error profile $\mathbf{E}^{*}$ of the coupled SE satisfies $\mathbf{E}^{*} \prec \mathbf{E}_{0}$.

Proof: Assume that, under these hypotheses, there exists a saturated profile $\mathbf{E}$ associated to $\mathbf{E}^{*}$ such that $\mathbf{E} \succ \mathbf{E}_{0}$. By Lemma 5.6 and the positivity of $\Delta F_{\mathrm{u}}$ as $R<R_{\mathrm{pot}}$, we have $\left|F_{\mathrm{c}}(\mathbf{E})-F_{\mathrm{c}}(\mathrm{S}(\mathbf{E}))\right| \geq \Delta F_{\mathrm{u}}$. Therefore, by Lemmas 5.2 and 5.4 we get $K / w>\Delta F_{\mathbf{u}} \Rightarrow w<K / \Delta F_{\mathrm{u}}$, a contradiction. Hence, $\mathbf{E} \preceq \mathbf{E}_{0}$. Since $\mathbf{E} \succ \mathbf{E}^{*}$ we have $\mathbf{E}^{*} \prec \mathbf{E}_{0}$.

Corollary 5.8: By first taking $\Gamma \rightarrow \infty$ and then $w \rightarrow \infty$, the AMP threshold of the coupled ensemble satisfies $R_{\mathrm{c}} \geq R_{\mathrm{pot}}$.

This result follows from Theorem 5.7 and Definition 3.4. It says that the AMP threshold for the coupled codes saturates the potential threshold. To prove it cannot surpass it requires a separate treatment. For our purposes this is not really needed because we have necessarily $R_{\mathrm{c}}<C$ and we know (Sec. III-C) that $\lim _{B \rightarrow \infty} R_{\text {pot }}=C$. Thus $\lim _{B \rightarrow \infty} R_{\mathrm{c}}=C$.

\section{ACKNOWLEDGMENTS}

J.B and M.D acknowledge funding from the Swiss National Science Foundation grant num. 200021-156672. We thank Marc Vuffray and Rüdiger Urbanke for discussions.

\section{REFERENCES}

[1] A. Barron and A. Joseph, "Toward fast reliable communication at rates near capacity with gaussian noise," in Information Theory Proceedings (ISIT), 2010 IEEE International Symposium on, June 2010, pp. 315-319.

[2] A. Joseph and A. R. Barron, "Fast sparse superposition codes have near exponential error probability for R<C," IEEE Transactions on Information Theory, vol. 60, no. 2, pp. 919-942, 2014.

[3] A. R. Barron and S. Cho, "High-rate sparse superposition codes with iteratively optimal estimates," in Information Theory Proceedings (ISIT), 2012 IEEE International Symposium on. IEEE, 2012, pp. 120-124.

[4] J. Barbier and F. Krzakala, "Replica analysis and approximate message passing decoder for superposition codes," in Information Theory Proceedings (ISIT), 2014 IEEE International Symposium on, 2014.

[5] C. Rush, A. Greig, and R. Venkataramanan, "Capacity-achieving sparse superposition codes via approximate message passing decoding," arXiv preprint arXiv:1501.05892, 2015.

[6] J. Barbier, C. Schülke, and F. Krzakala, "Approximate message-passing with spatially coupled structured operators, with applications to compressed sensing and sparse superposition codes," Journal of Statistical Mechanics: Theory and Experiment, vol. 2015, no. 5, 2015.

[7] J. Barbier and F. Krzakala, "Approximate message-passing decoder and capacity-achieving sparse superposition codes," 2015. [Online]. Available: http://arxiv.org/abs/1503.08040

[8] S. Kudekar, T. Richardson, and R. Urbanke, "Spatially coupled ensembles universally achieve capacity under belief propagation," Information Theory, IEEE Transactions on, vol. 59, no. 12, pp. 7761-7813, 2013.

[9] M. Lentmaier, A. Sridharan, J. Costello, D.J., and K. Zigangirov, "Iterative decoding threshold analysis for ldpc convolutional codes," Information Theory, IEEE Transactions on, vol. 56, no. 10, pp. 52745289, 2010

[10] A. Javanmard and A. Montanari, "State evolution for general approximate message passing algorithms, with applications to spatial coupling," Journal of Information and Inference, vol. 2, no. 2, pp. 115-144, 2013.

[11] A. Yedla, Y.-Y. Jian, P. S. Nguyen, and H. Pfister, "A simple proof of threshold saturation for coupled scalar recursions," in 7th International Symposium on Turbo Codes and Iterative Information Processing (ISTC), 2012, pp. 51-55.

[12] S. Kumar, A. J. Young, N. Macris, and H. Pfister, "Threshold saturation for spatially-coupled ldpc and ldgm codes on bms channels," IEEE Transactions on Information Theory, vol. 60, pp. 7389-7415, 2013.

[13] M. Bayati and A. Montanari, "The dynamics of message passing on dense graphs, with applications to compressed sensing," IEEE Transactions on Information Theory, vol. 57, no. 2, pp. 764 -785, 2011.

[14] A. Yedla, Y.-Y. Jian, P. Nguyen, and H. Pfister, "A simple proof of maxwell saturation for coupled scalar recursions," Information Theory, IEEE Transactions on, vol. 60, no. 11, pp. 6943-6965, 2014.

[15] F. Krzakala, M. Mézard, F. Sausset, Y. Sun, and L. Zdeborová, "Probabilistic reconstruction in compressed sensing: Algorithms, phase diagrams, and threshold achieving matrices," Journal of Statistical Mechanics: Theory and Experiment, vol. P08009, 2012.

[16] F. Caltagirone and L. Zdeborová, "Properties of spatial coupling in compressed sensing," CoRR, vol. abs/1401.6380, 2014.

[17] D. Guo, Y. Wu, S. Shamai, and S. Verdú, "Estimation in gaussian noise: Properties of the minimum mean-square error," Information Theory, IEEE Transactions on, vol. 57, no. 4, pp. 2371-2385, 2011.

[18] J. Barbier, "Statistical physics and approximate message-passing algorithms for sparse linear estimation problems in signal processing and coding theory," Ph.D. dissertation, Université Paris Diderot, 2015. [Online]. Available: http://arxiv.org/abs/1511.01650

[19] A. Joseph and A. R. Barron, "Least squares superposition codes of moderate dictionary size are reliable at rates up to capacity," IEEE Transactions on Information Theory, vol. 58, no. 5, pp. 2541-2557, 2012. 